\title{
Multi-purpose regridding in vortex methods
}

\author{
G.-H. Cottet, M.-L. Ould Salihi and M. El Hamraoui \\ LMC-IMAG, Université Joseph Fourier, Grenoble, France
}

\begin{abstract}
This paper is concerned by regridding strategies for accurate vortex calculations. We first demonstrate on several test cases of two and three dimensional, inviscid as well as viscous flows, that, when based on high order interpolation formulas, these technics do allow to maintain accuracy for long time simulations. We then show that, by alternating regridding on several non-conforming grids, one can easily design particle-based domain decomposition algorithms. This is illustrated by the example of a flow past two cylinders.
\end{abstract}

\section{Introduction}

A common difficulty to all Lagrangian methods is in handling severe grid distortions. In vortex methods, particles move with the flow and carry circulations. These particles describe the flow in a consistent way only when averaged over a scale which is determined by the desired resolution. Each particle is the center of a blob of finite size and convergence analysis as well as numerical experiments show that these blobs must maintain a certain amount of overlapping to provide an accurate solution. In most flows of interests, this overlap is severely affected by velocity gradients. A typical situation is around a stagnation point, where particles would tend to cluster in some directions and to spread along the other directions.

In these situations, the theoretical high order of convergence cannot be observed in practice. Error estimates actually allow to understand the mechanisms through which accuracy deteriorates. One may consider two types of error analysis for particle methods. A first class only requires the flow to be divergence free and leads to error estimates of Monte-Carlo type with low convergence rates, but independent of time. In this case particles are initialized randomly. In the second class, particles are initialized at collocation points corresponding to second or higher order quadrature rules, and high order error estimates can derived but with constants which grow exponentially in time at a rate depending on the velocity gradients. In presence of strong shear, one observes that, as time goes on, the accuracy of the method degenerates from that expected in the second type of estimates to the low order accuracy behavior predicted in the first type of analysis.

Several recipes have been proposed to maintain high order accuracy for longer times. One is to process the circulations of the particles to compensate for the lack of overlapping of the blobs. This can be done essentially in two ways. One may for instance require the particle circulations to allow to recover the actual vorticity value at the center of the blobs. This consistency check leads to a system of linear equations to solve, in general through an iterative algorithm (Beale, 1981). In another approach, reminiscent to adaptive quadrature technics, particles are assigned volumes corresponding to quadrature weights which enforce that numerical integration is exact for polynomials up to a given degree (Strain, 1997). 
The efficiency of these techniques is however limited to flow which retain a minimal smoothness, so that particles do not go too far apart. For more singular flows, in particular in three dimensions when the strain in the flow goes with vorticity intensification, it may become necessary to restart particles at regular locations. In some important particular cases (for instance vortex sheets or 3D jets), the connectivity of the computational elements can be tracked, and regridding can be simply and accurately achieved by inserting new elements between connected particles when their distance exceeds a certain value. In general however, and in particular for viscous flows, it is difficult to track the connectivity of the elements and regridding requires to restart particles from scratch on a regular grid. The circulations on the restarted particles must be obtained by classical interpolation formulas. This has the drawback to rely in an intermediate stage on an Eulerian grid, but only an integer pointer needs to be attached to this grid. Regridding has been in the recent years an essential ingredient for high resolution vortex calculations (we refer for instance to Koumoutsakos (1997) for inviscid ellipse simulations).

A remarkable feature of regridding is that it allows to maintain the overlap of the particles even when they are surrounded by blobs of variable sizes. Variable blobs can be of great interests when the flow scales are not uniform throughout the computational domain. In the context of domain decomposition, regridding also allows to transfer in a simple and consistent way vorticity between particle distributions associated to different sub-domains.

The goal of this paper is to illustrate these features and to show that, when implemented with high order interpolation formulas, regridding enables accurate two or three dimensional viscous flow calculations. In the next section we review the tools to construct and analyze interpolation formulas in the context of regridding. We give some numerical illustrations on the basis of which we were able to choose an interpolation kernel which combines accuracy and smoothness.

In section 3, we focus on three examples where particle regridding is essential to achieve accuracy at a minimal computational cost : a three dimensional ring impinging a wall, the wake behind a cylinder, and the wake behind two asymmetric cylinders.

\section{Interpolation kernels for high order regridding}

A natural way to control the accuracy of regridding is by enforcing that the original and remeshed particles share the same total circulation, linear impulse, angular impulse, and so on. This yields a hierarchy of regridding schemes. Fourier analysis shows that these requirements can be translated into the order of accuracy of the method (respectively first, second and third order for the invariants above mentioned).

In practice, interpolation formulas are most often obtained by tensor products of one-dimensional formulas. To simplify the notations we may thus focus one one dimensional formulas. We will also consider the case when the vorticity is a scalar. Let us denote by $\left(\tilde{x}_{p}\right)$ and $\left(x_{p}\right)$ respectively the original and remeshed locations, and by $\tilde{\omega}_{p}, \tilde{\Gamma}_{p}, \omega_{p}$ and $\Gamma_{p}$ the associated local vorticity values and circulations. The conservation of the three first invariants (circulation, linear and angular impulse) reads

$$
\begin{aligned}
\sum_{p} \Gamma_{p} & =\sum_{p} \tilde{\Gamma}_{p} \\
\sum_{p} x_{p} \Gamma_{p} & =\sum_{p} \tilde{x}_{p} \tilde{\Gamma}_{p}
\end{aligned}
$$




$$
\sum_{p}\left|x_{p}\right|^{2} \Gamma_{p}=\sum_{p}\left|\tilde{x}_{p}\right|^{2} \tilde{\Gamma}_{p}
$$

The circulations $\Gamma_{p}$ are most easily recovered through interpolation. Assume the new particles are on a uniform rectilinear grid of grid-size $h$. Given an interpolation kernel $W$, the new circulations are then computed by the formula

$$
\Gamma_{p}=\sum_{q} \tilde{\Gamma}_{q} W\left(\frac{x_{p}-\tilde{x}_{q}}{h}\right)
$$

and the above conservation properties lead to the following conditions:

$$
\begin{aligned}
\sum_{q} W\left(\frac{x-x_{p}}{h}\right) & \equiv 1 \\
\sum_{q}\left(x-x_{q}\right) W\left(\frac{x-x_{q}}{h}\right) & \equiv 0 \\
\sum_{q}\left|x-x_{q}\right|^{2} W\left(\frac{x-x_{q}}{h}\right) & \equiv 0
\end{aligned}
$$

This can be viewed as a linear system for the quantities $W\left(\frac{x-x_{p}}{h}\right)$, the solution of which requires the right number of points ( 3 for three invariants) and yields a piecewise polynomial function $W$. In the case under consideration one obtains the following piecewise quadratic function

$$
\Lambda_{2}(x)= \begin{cases}1-x^{2}, & \text { if } 0 \leq x<1 / 2 \\ (1-x)(2-x) / 2, & \text { if } 1 / 2 \leq x<3 / 2 \\ 0, & \text { otherwise }\end{cases}
$$

This interpolation kernel leads to a third order interpolation formula. Note that requiring only the 2 first invariants to be conserved would lead to a second order formula (the classical piecewise linear "tent" function) which might be thought sufficiently accurate. However practice dictates that, in order to avoid grid distortions which might prevent from recovering a smooth field, regridding must be done frequently. A second order formula then turns out to be too dissipative and higher order formulas are necessary.

Although it has been used successfully in a number of high resolution calculations, an obvious drawback of the $\Lambda_{2}$ formula is its lack of smoothness (it is not even continuous). If not used frequently enough, this kernel may amplify small unphysical oscillations. Smoother functions can be found in two different ways. One can first require the conservation of additional invariants. The following piecewise cubic formula has a support which contains five points and conserves the four first invariants:

$$
\Lambda_{3}(x)= \begin{cases}\left(1-x^{2}\right)(2-x) / 2, & \text { if } 0 \leq x<1 \\ (1-x)(2-x)(3-x) / 6, & \text { if } 1 \leq x<2 \\ 0, & \text { otherwise }\end{cases}
$$

This function is continuous. An alternative approach is reminiscent to a classical construction of high order cut-off from low order ones: one starts from a smooth second order kernel $W$, and combines it with its derivative to satisfy the next invariant. The modified kernel is given by the formula

$$
\tilde{W}(x)=\frac{1}{2}\left(3 W+x W^{\prime}\right)
$$




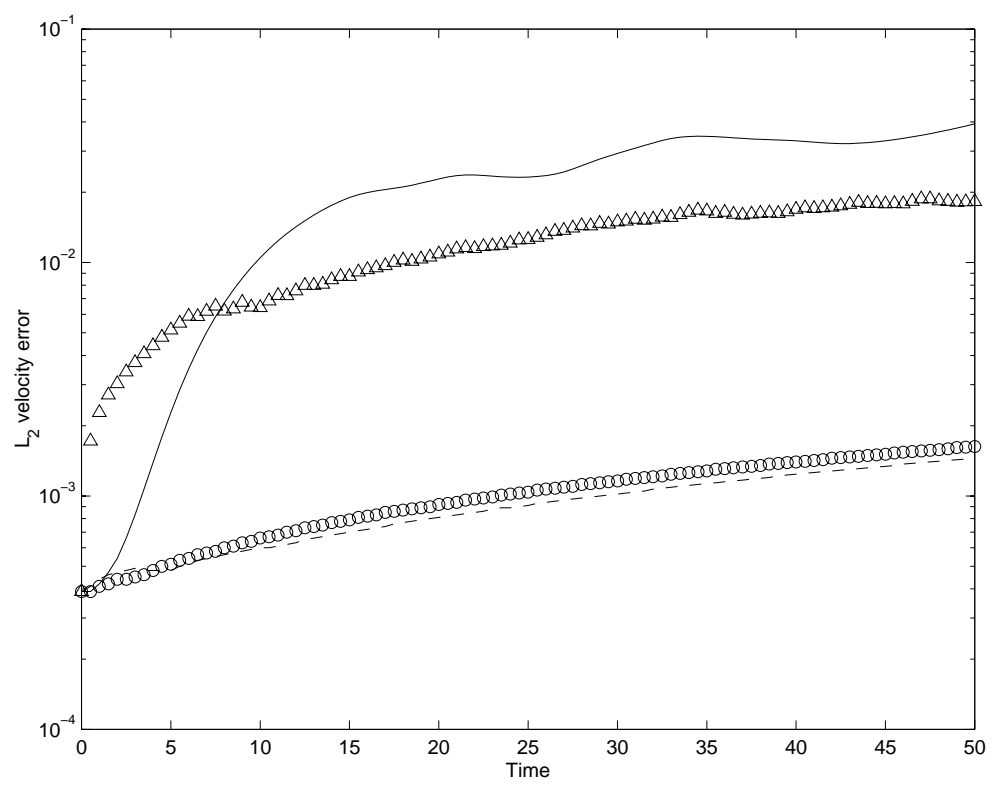

Figure 1: Error curves for $\omega_{0}(x)=\left(1-|x|^{2}\right)^{3} ; h=.05$. No-remesh, (solid curve), remeshing $\Lambda_{2}$ $(\triangle)$, remeshing $\Lambda_{3}(\bigcirc)$ and remeshing $M_{4}^{\prime}$ (dotted curve).

In the case when $W$ is cubic spline (that is the $C^{2}$ function obtained by two successive convolutions of the top-hat function by itself), this gives the following kernel, first suggested by Monaghan (1985) in the context of SPH methods

$$
M_{4}^{\prime}(x)= \begin{cases}0 & \text { if }|x|>2 \\ \frac{1}{2}(2-|x|)^{2}(1-|x|) & \text { if } 1 \leq|x| \leq 2 \\ 1-\frac{5 x^{2}}{2}+\frac{3|x|^{3}}{2} & \text { if }|x| \leq 1\end{cases}
$$

This is $C^{1}$ function. As $\Lambda_{3}$, it extends to the four nearest particles (in three dimensions this implies a stencil of 64 points).

The respective efficiency of these kernels has been tested on the classical case of an axisymmetric vortex patch. This particular vorticity field gives a steady solution to the Euler equations but particles may move along circles at different speed, thus inducing a strain in their distribution. This strain is enough to deteriorate the accuracy of vortex method if no processing is done on either the circulations or the locations of the particles. In Fig. 1 we have plotted the $L^{2}$ velocity errors with the no-remeshed original method and with the remeshing $\Lambda_{2}, \Lambda_{3}$ and $M_{4}^{\prime}$. When noremeshing is done the accuracy rapidly grows and saturates at a level which one may conjecture as corresponding to a random initialization case. Remeshing with $\Lambda_{2}$ slightly improves the results for large times. When $\Lambda_{3}$ or $M_{4}^{\prime}$ are used, the results are one order of magnitude better. Note that in these calculations, remeshing was done at every time-step. Regridding less frequently does not modify the results, provided it does not give time to the flow for producing too much distortion. High order and smooth regridding enables the particles to essentially retain their initial accuracy. Note that a smaller value of the initial particle spacing would give even more dramatic improvements (the error for the no-remeshed calculation would reach about the same value while the remeshed calculations would gain for an other order of magnitude in accuracy). 


\section{Remeshing for accurate viscous flow calculations}

A conclusion of the above discussion is that the kernel $M_{4}^{\prime}$ presents a good combination of smoothness and accuracy. All the calculations presented below have been done with the regridding scheme based on this interpolation.

\subsection{Vortex wall interaction}

Our first example is the simulation of a vortex ring impinging on a wall at an angle of 30 degrees. The Reynolds number of 1400 allows comparisons with the experiments of Lim (1989) and the finite-difference results reported by Verzicco and Orlandi (1994).

Particles are initialized in a box $[0,1] \times[0,1] \times[0,1 / 2]$. The boundary conditions are periodic in the $x$ and $y$-directions. No-slip in enforced at $z=0$, and at $z=.5$ no-through flow and zero vorticity are assumed. Diffusion is solved by a Particle Strength Exchange scheme and the velocity fast calculation is done on an Eulerian grid by a Vortex-In-Cell scheme, using the $M_{4}^{\prime}$ function to transfer vorticity onto the grid and interpolate the velocity and the stretching back onto the particles. Finally the no-slip condition is translated into vorticity flux conditions which enforce the solenoidal condition at the wall (we refer to Cottet and Koumoutsakos (1999) for details).

Regridding is essential here to respond to the wall production of vorticity, vorticity stretching and vorticity expansion through diffusion. Particles are initialized in the support of the ring on a $128^{3}$ uniform grid (which gives a resolution comparable to the one used in Verzicco and Orlandi (1994)). This results in about 600,000 particles. At the time of the calculation showed in Fig.2 this number has increased to slightly less than 1 million, which means that particles occupy about half of the computational domain. The bottom picture represents the isosurface of vorticity magnitude, corresponding to $40 \%$ of its maximum value, colored by the $y$-component of the vorticity. The bottom picture represents the contours of this component. The three-dimensionality of the flow is apparent in the pumping of the vorticity from the lower part of the ring, through stretching, to the upper part which gains enough circulation to rebound from the wall. These results are in very close agreement with those reported by Verzicco and Orlandi (1994). Animations of this flow and others can be downloaded from the web-site http://www-lmc.imag.fr/lmc-edp/Georges-Henri. Cottet/vortex.html.

It should be emphasized that in this calculation regridding is done at every time-step. A close examination of the energy decay shows that remeshing produces no discernable extra dissipation. However it is plausible that in turbulent flows remeshing would act at a sub-grid level. Turbulence experiments which quantify the related subgrid-scale dissipation are reported elsewhere (Cottet, Ossia, Vanderlinden, 1999).

\subsection{Variable-blob methods for wake calculations}

In bluff-body flows the relevant scales can widely vary from one part of the flow to another. For optimal results this has to be taken into account in the implementation of a given numerical scheme. In that respect vortex methods are no different from any numerical method based on an Eulerian grid. 
In the context of vortex methods variable scales are clearly best resolved by variable-size blobs. Vortex methods with variable blobs can be designed in a consistent way for both ideal and viscous flows. While the advection of particles is straightforward and only relies on the Biot-Savart law mollified on a variable scale, diffusion with a PSE scheme requires a mapping of the variable blobs onto uniform blobs. PSE formulas are then applied to the modified diffusion operator in the mapped coordinates (see Cottet and Koumoutsakos (1999) or Cottet, Koumoutsakos and Ould Salihi (1999) for details). The overlapping of the blobs requires that the particle density is always and everywhere coherent with the blob sizes and periodic remeshing is the only way to enforce this property.

The case of a wake behind a cylinder is a simple but illustrative example. In the flow depicted in Fig.3 the blob size is scaled by the distance to the center of the cylinder. The grid on which particles are initialized and remeshed is a polar grid with exponential stretching in the radial direction. This effectively provides particles with volumes increasing like $r^{2}$, in accordance with the chosen blob-sizes. Regridding is necessary in order to avoid particles with large volumes to reach zones of the flow with small blob-sizes.

The Reynolds number is 150 and the number of particles at the end of the simulation is below 10,000 , which, given the extent of the wake, is fairly small. A close inspection of the drag curve indicates a Strouhal frequency of about .183 and a mean drag value of 1.43 , in very good agreement with the experimental results of Williamson (1991). This method clearly allows a fine resolution of the vorticity near the boundary at a minimal cost. It has been successfully used in the simulation of the open loop control strategies based on periodic rotations of the cylinder. More numerical illustrations are given in Cottet, Koumoutsakos and Ould Salihi (1999).

\subsection{Domain decomposition methods for flows around multiple bodies}

Domain decomposition is a classical tool for flow simulations in complex geometries. It in particular allows the use of different grid resolutions in different parts of the flow. A key ingredient in all these methods is the definition of consistent interface conditions. For the Navier-Stokes equations in velocity-vorticity formulation, these conditions are needed at two levels: for the vorticity unsteady equation and for the velocity calculation in terms of the vorticity.

Assuming that the domains overlap, the later one may be dealt with through the Schwarz alternating method. For the vorticity equation, regridding gives an elegant way to ensure the correct transfer of vorticity between the sub-domains. To be more specific, consider the case of 2 domains $\Omega_{1}$ and $\Omega_{2}$, each of them discretized by a particle distribution corresponding to a given mapping (for instance curvilinear coordinates around an obstacle and a uniform cartesian grid elsewhere). It is classical to view these distributions as collocation points corresponding to a given quadrature formula. Assume that at a time level $t_{n}$ the vorticity equation has been advanced in a first sweep in $\Omega_{1}$. To update the solution in $\Omega_{2}$, one needs the vorticity in a neighborhood of the boundary $\partial \Omega_{2}$ of $\Omega_{2}$. This may be obtained from the solution just computed in $\Omega_{1}$. However, the vorticity field in $\Omega_{1}$ is known on particles associated to the resolution defined in this domain. An accurate solution in $\Omega_{2}$ requires particle locations corresponding to the quadrature rule chosen in $\Omega_{2}$. One thus have to remesh particles in the overlapping zone onto theses locations, and compute the resulting circulations. In other words, interface conditions are realized by mapping alternatively particles lying in the overlapping zone onto locations consistent with the resolution enforced in the sub-domains. Obviously, it is here of crucial importance to use in these successive remeshings 
interpolation rules of high accuracy.

As an example, Fig. 6 shows the evolution of the flow past 2 asymmetric cylinders. In this case we have used 3 meshes: 1 polar grid around each cylinder, and 1 cartesian grid in between, as shown on the top picture. The total number of particles at the last stage of the computation is about 100, 000. The bottom pictures show the evolution of the vorticity. In particular it illustrates that the interface conditions enable a smooth transition between the sub-domains.

\section{References}

1 BeAle, J.T.: On the accuracy of vortex methods for large times. In "Computational Fluid Dynamics and Reacting Gas Flows", B. Engquist et al., eds, Springer-Verlag, New-York, 19-32.

2 Cottet, G.-H., and Koumoutsakos, P.: Vortex methods. Cambridge University Press, to appear.

3 Cottet, G.-H., Ossia, S. And Vanderlinden, G.: Performance of vortex methods in the simulation of homogeneous isotropic turbulence, submitted, 1999.

4 Cottet, G.-H., Koumoutsakos, P. and Ould Sahili, M.L.: Towards adaptive vortex methods. In preparation.

5 Koumoutsakos, P.: Inviscid Axisymmetrization of an Elliptical Vortex. J. Comp. Phys. 138 (1997), 821-857.

6 LiM, T.T.: An experimental study of a vortex ring interacting with an inclined wall. Exp. in Fluids 6 (1989), 453-463.

7 Monaghan, J.J.: Extrapolating B-splines for interpolation. J. Comp. Phys. 60 (1985), 253-262.

8 Ould Sahili, M.L., Cottet, G-H. and El Hamraoui, M.: Blending finite-difference and vortex methods for incompressible flow simulations. Submitted.

9 Strain, J.A.: Fast adaptive 2D vortex methods. J. Comp. Phys. 132 (1997), 108-122.

10 Verzicco, R., AND Orlandi, P.: Normal and oblique collision of a vortex ring with a wall. Meccanica 29 (1994), 383-391.

11 Williamson, C.H.K: 2D and 3D aspects of the wake of a cylinder and their relation with wake computation. Lectures in Applied Math. 28 (1991), 719-751. 

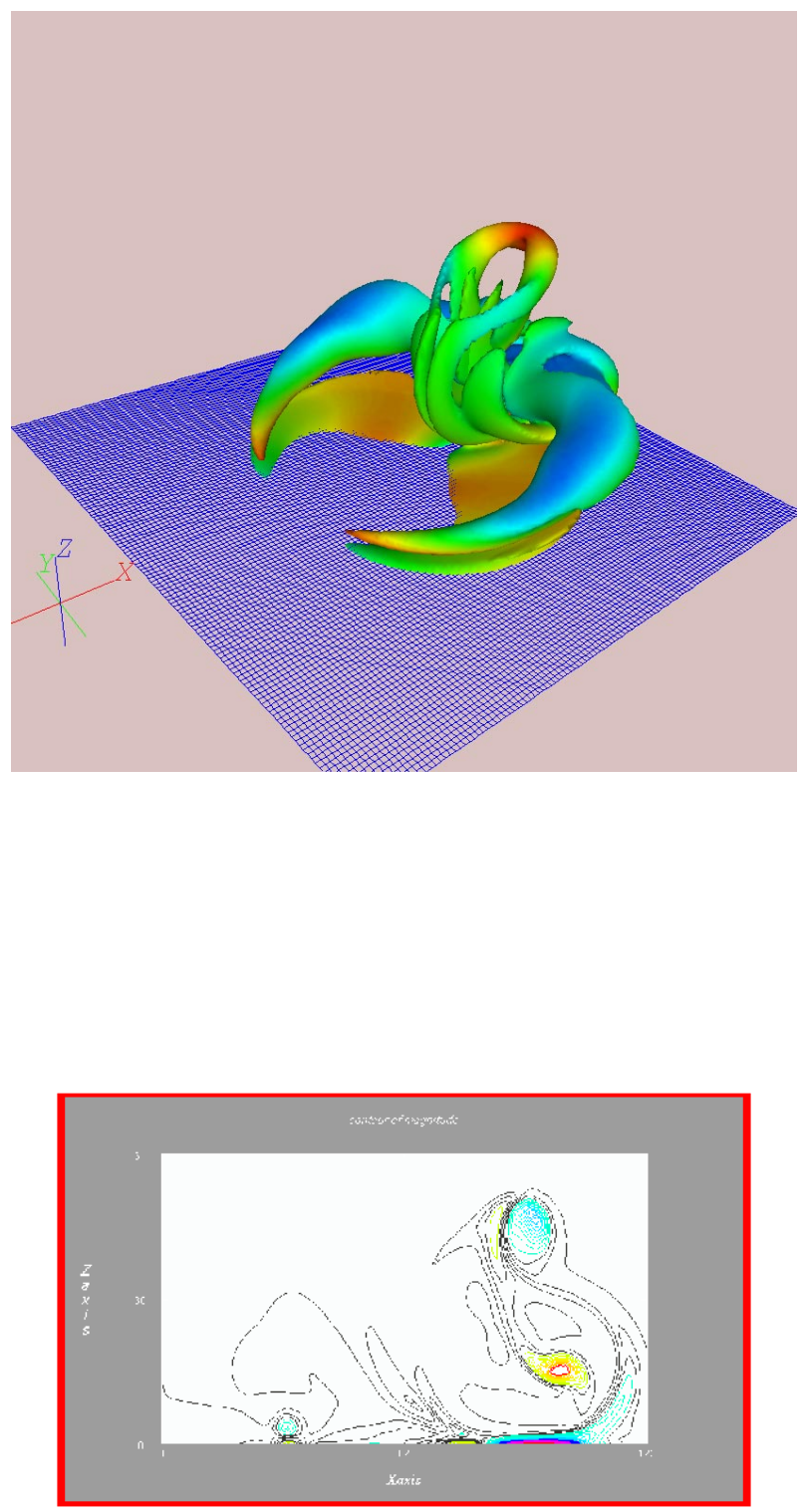

Figure 2: Rebound of a vortex ring impinging a wall. Isosurface of vorticity magnitude colored by the $y$-vorticity component and contours of this component. 


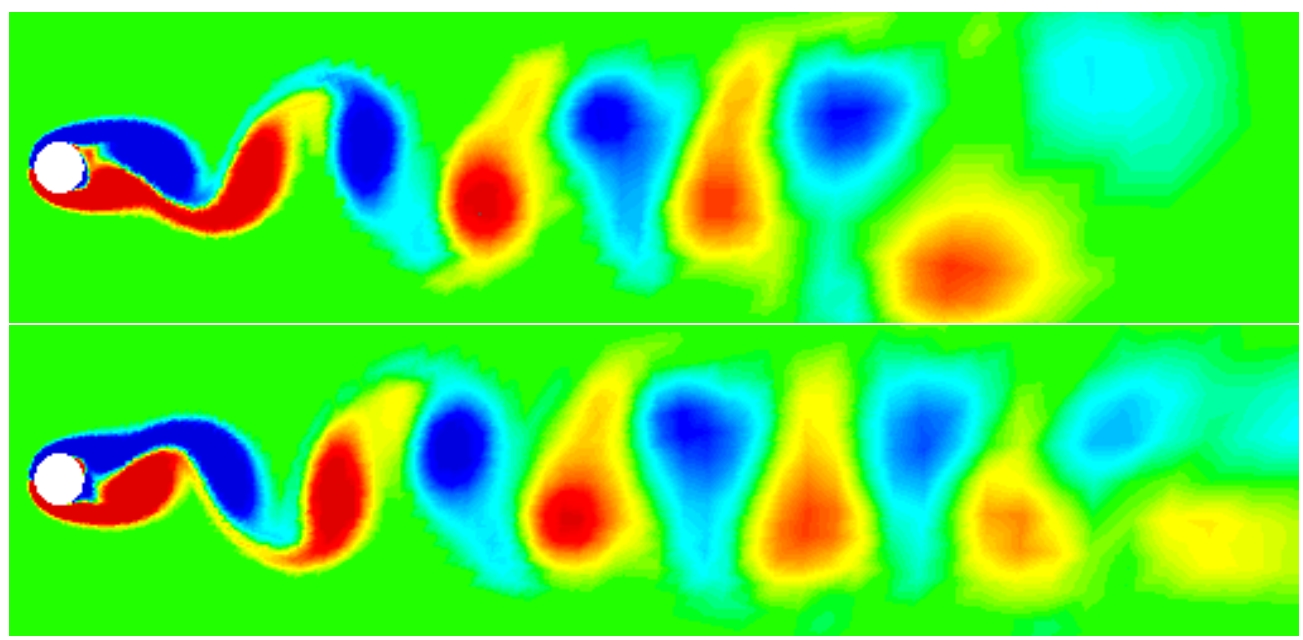

Figure 3: Vortex shedding behind a cylinder with a variable-blob method. Top picture : $T=66$; bottom picture : $T=114$
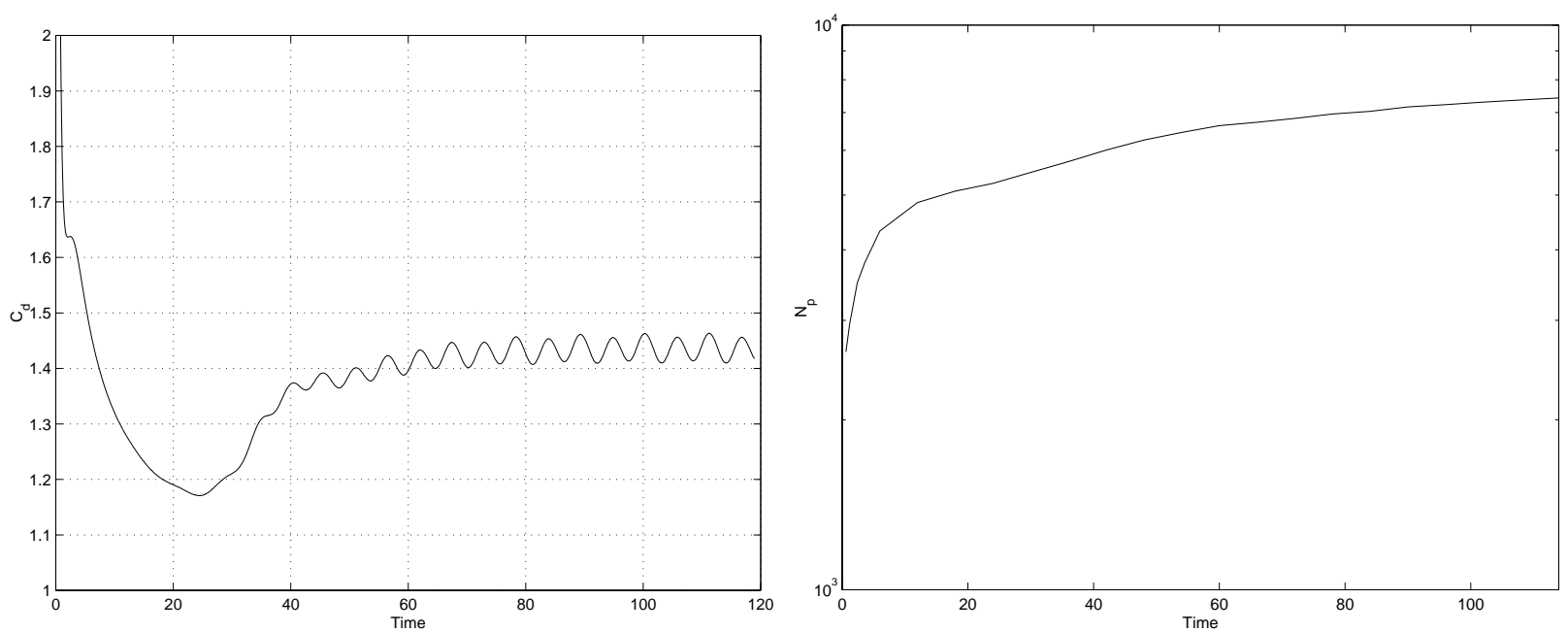

Figure 4: Vortex shedding behind a cylinder with a variable-blob method. Left picture : number of particles versus time; right picture : drag coefficient versus time 


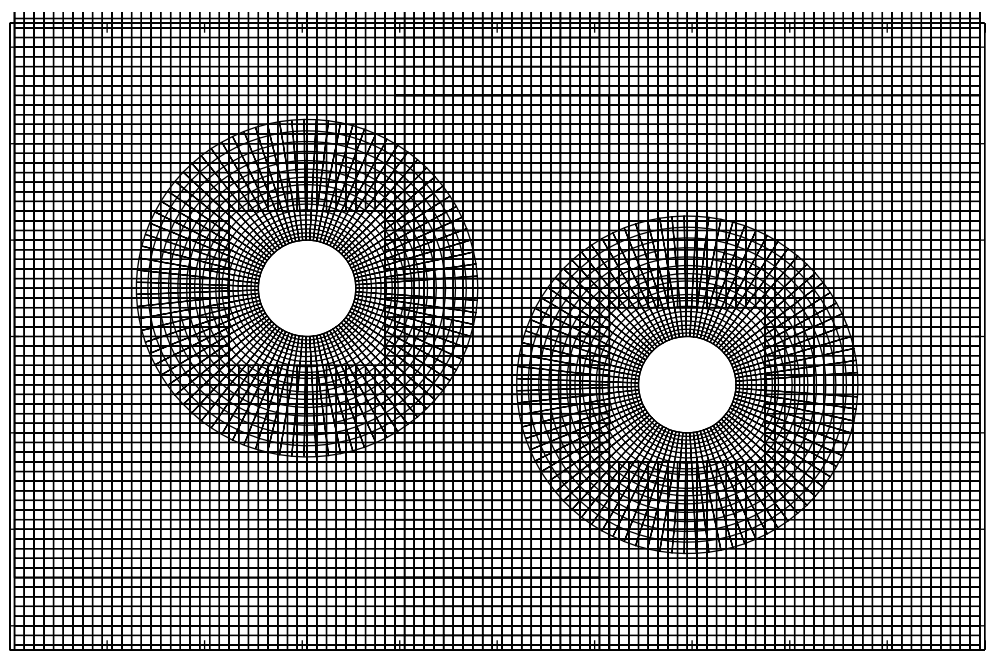

Figure 5: Flow around 2 asymmetric cylinders. Grids used for regridding the particles
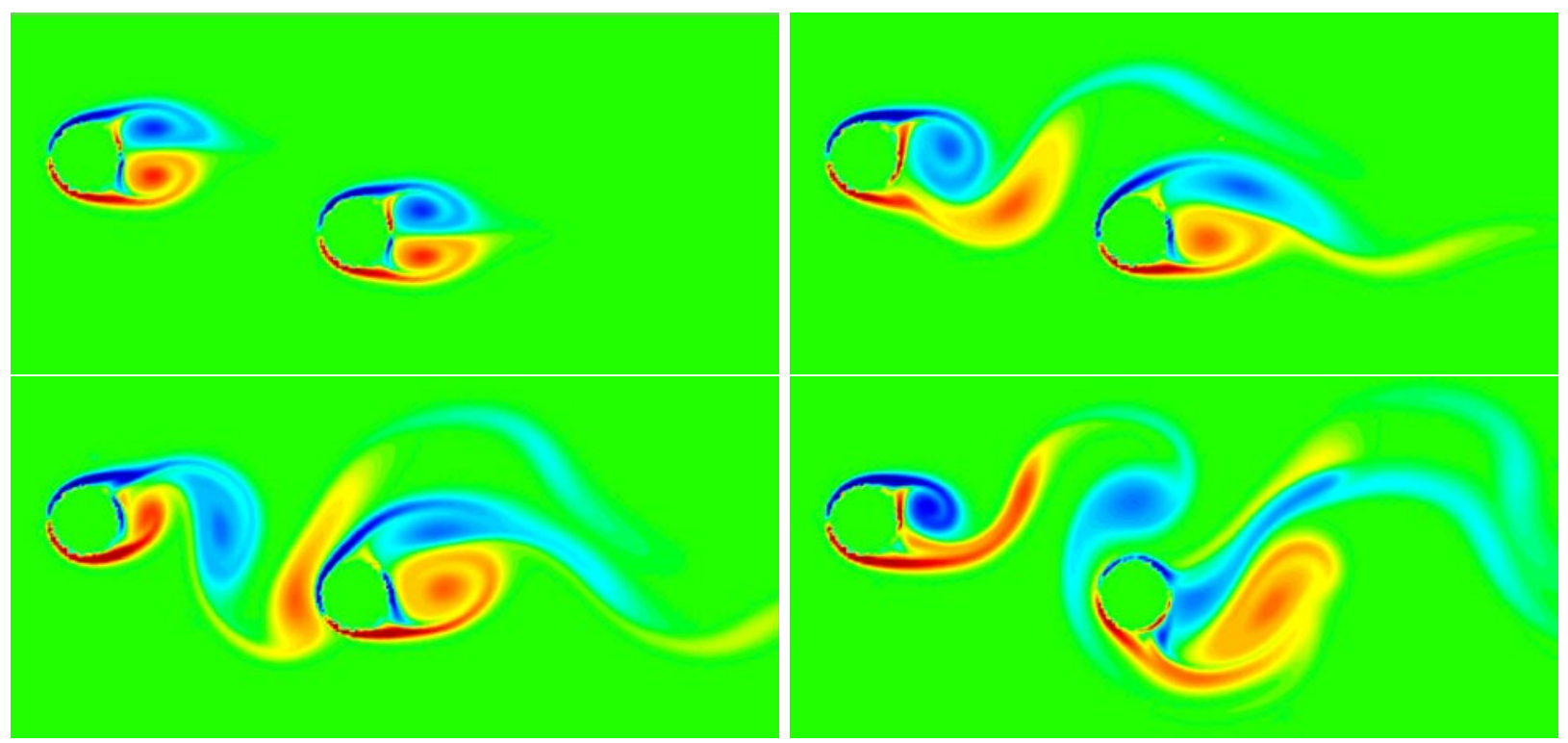

Figure 6: Flow around 2 asymmetric cylinders. Successive stages of the vorticity contours 